\title{
HIPERTENSÃO PULMONAR EM CÃES: ARTIGO DE REVISÃO
}

\author{
Raquel de Araujo Cantarella ${ }^{1}$, Taís Marchand Moreira Rocha ${ }^{1}$, \\ Marcela Ferreira Terres ${ }^{1}$, Ana Laura Angeli ${ }^{1}$ \\ 1 Universidade Tuiuti do Paraná - raquelcantarella@hotmail.com
}

RESUMO: A Hipertensão Arterial Pulmonar (HAP) ou Hipertensão Pulmonar (HP) é uma doença progressiva, ainda pouco descrita na medicina veterinária, caracterizada por aumento da resistência vascular pulmonar e conseqüente aumento da pressão arterial pulmonar, resultando principalmente em disfunção ventricular direita e morte por insuficiência cardíaca na ausência de tratamento. $\mathrm{Na}$ medicina veterinária a HP geralmente é identificada durante a avaliação de animais com doença pulmonar crônica ou insuficiência cardíaca direita. Portanto, o risco de pacientes veterinários apresentarem HP é refletido pela probabilidade de apresentarem doenças pulmonares ou valvares crônicas. O diagnóstico é realizado por ecocardiografia Doppler ou cateterismo cardíaco e o tratamento visa a eliminação da causa primária e estabilização cardiovascular, melhora na oxigenação e redução da pressão na artéria pulmonar.

Palavras-chave: cães; disfunção ventricular; hipertensão arterial pulmonar

\section{PULMONARY HYPERTENSION IN DOGS: REVIEW ARTICLE}

\begin{abstract}
Pulmonary Arterial Hypertension (PAH) or Pulmonary Hypertension (PH) is a progressive disease, poorly described in Veterinary Medicine, which is characterized by increased pulmonary vascular resistance and consequently increased pulmonary arterial pressure. This situation ends with progressive right ventricular dysfunction and death from heart failure if untreated. In Veterinary Medicine, PH normally is identified during the evaluation of animals with chronic pulmonary disease or right cardiac failure. Hence, the risk of veterinary patients show $\mathrm{PH}$ is reflected by the probability that they have to suffer from pulmonary or chronic cardiac valve diseases. The diagnostic is performed by Doppler echography or cardiac catheterism and treatment is based on primary cause elimination and cardiovascular stabilization, as well as oxygen improvement and pulmonary arterial pressure reduction.
\end{abstract}

Key words: dogs; pulmonary arterial hypertension; right ventricular dysfunction 


\section{INTRODUÇÃO}

A Hipertensão Arterial Pulmonar (HAP) ou Hipertensão Pulmonar (HP) é caracterizada pela presença de vasoconstrição pulmonar, trombose in situ e remodelamento vascular que pode levar principalmente à insuficiência ventricular direita progressiva e óbito (O'Callaghan e Gaine, 2004; Lapa et al., 2006). Belerenian (2003) denomina cor pulmonale o quadro de hipertensão pulmonar que altera a estrutura e função do ventrículo direito, de forma secundária à alteração do parênquima e/ou da vascularização pulmonar.

Como parte integrante da circulação central, a circulação pulmonar é um sistema de baixa pressão ou baixa resistência vascular. Os leitos vasculares pulmonar apresentam grande complacência, permitindo a acomodação de grande quantidade de volume sanguíneo e contribuem para a redução da pressão arterial local. Assim, a baixa pressão no circuito pulmonar favorece o trabalho do ventrículo direito e a irrigação miocárdica pela coronária direita. Sabese que a pressão sistólica na artéria pulmonar em um cão sadio é de 15$25 \mathrm{mmHg}$, a diastólica de $5-10 \mathrm{mmHg}$ e a média de $10-15 \mathrm{mmHg}$ (Belerenian, 2003).

\section{ETIOPATOGENIA E EPIDEMIOLOGIA}

Em um estudo feito por Kellum e Stepien (2007) utilizando 22 cães com HP, realizou-se uma estimativa da pressão arterial sistólica pulmonar/ ventrículo direito determinada por exame ecocardiográfico onde, através desta estimativa, os cães foram classificados como portadores de HP leve $(31-50 \mathrm{~mm} / \mathrm{Hg})$, moderada (51 $75 \mathrm{~mm} / \mathrm{Hg}$ ) ou severa (acima de $75 \mathrm{~mm} / \mathrm{Hg}$ ).

Segundo Zabka e colaboradores (2006) a HPA deve ser subdividida em cinco classes: idiopática; familiar; associada a fatores de risco como doenças do colágeno, hipertensão portal, shunts congênitos sistêmicopulmonares e hemoglobinopatias; associado a pacientes esplenectomizados; hipertensão pulmonar persistente do recém-nascido; e por envolvimento capilar ou venoso, como nas doenças pulmonares veno-oclusivas e hemangiomatose capilar pulmonar. Belerenian (2003) descreve que a HP na medicina veterinária pode ser classificada em primária; secundária a tromboembolia pulmonar, doenças cardíacas e doenças respiratórias; ou idiopática. Porém, Lapa e colaboradores (2006) afirmam que a HP somente pode ser classificada em hipertensão arterial pulmonar idiopática quando nenhum fator causal pode ser identificado.

Hawkins (2004) descreve que a HP é quase sempre secundária a doenças pré ou pós-capilares na medicina veterinária. As causas précapilares incluem doença pulmonar e doença da altitude elevada, as quais provocam vasoconstrição local e hipóxia alveolar; doença cardíaca congênita que causa derivações da esquerda para direita (ducto arterioso patente $\mathrm{e}$ defeitos septais), resultando em aumento do fluxo sangüíneo pulmonar; e tromboembolismo e vasculite pulmonar causada principalmente pela dirofilariose. As causas pós-capilares resultam do aumento da pressão venosa pulmonar ou atrial esquerda em decorrência da insuficiência ventricular esquerda, insuficiência ou estenose mitral, trombos ou neoplasias atriais esquerdas e obstrução venosa pulmonar.

O estudo realizado por Pyle e colaboradores (2004) utilizando 54 cães com HP, observou que a HP associada à insuficiência cardíaca esquerda tende a ser leve, porém cães com doença vascular pulmonar grave podem apresentar um aumento de 4 a 5 vezes 
da pressão no ventrículo direito e na artéria pulmonar. Assim, este estudo demonstrou que os cães classificados como HP leve a moderada apresentavam principalmente problema valvar crônico, porém os cães classificados como HP severa apresentavam principalmente doença respiratória associada. Neste mesmo estudo, $24 \%$ da população de cães avaliados não possuíam raça definida e $76 \%$ eram de raça pura, variando de 4 meses de idade a 17 anos (média de 10,5 anos). Dos cães incluídos na pesquisa, 39 animais apresentavam no mínimo 10 anos de idade, sendo 11 destes com aproximadamente 13 anos.

Segundo Morais (2008) e Kellum e Stepien (2007) os pacientes caninos são mais acometidos do que os felinos e as fêmeas idosas mais acometidas que os machos, sendo a maioria de pequeno porte. Zabka e colaboradores (2006) descreveram a HP em uma fêmea mestiça de chihuahua e um welsh corgi, ambos com persistência de ducto arterioso (PDA); e em dois animais da raça labrador, um com defeito interventricular e outro sem etiologia conhecida. Também foi descrito por Sarraff-Lopes e colaboradores (2006) um caso de hipertensão pulmonar primária em Yorkshire, macho, 3 anos de idade com histórico de episódios de síncope após esforço físico.

\section{PATOGENIA}

Para um cão em repouso, a pressão arterial pulmonar média é $13 \mathrm{mmHg}$, a pressão venosa pulmonar média é $5 \mathrm{mmHg}$ e o débito cardíaco é 2,5L/minuto. Assim, a resistência pulmonar (calculada através da diferença da pressão arterial pulmonar em relação à pressão venosa pulmonar) é $3,2 \mathrm{mmHg} / \mathrm{L} /$ minuto, valor equivalente à $1 / 12$ da resistência da circulação sistêmica. Assim, a baixa resistência na circulação sanguínea pulmonar em relação à circulação sanguínea sistêmica permite que a vascularização pulmonar receba grande volume sanguíneo. Esta complacência dos vasos sangüíneos pulmonares permite sua distensão vascular conforme 0 aumento do débito cardíaco, oferecendo menor resistência à circulação do fluxo pulmonar e favorecendo a hematose. Outro fator que influencia a vasomoção pulmonar é a concentração local de oxigênio, medida como pressão parcial de oxigênio $\left(\mathrm{PO}_{2}\right)$, sendo que uma diminuição da $\mathrm{PO}_{2}$ (hipóxia) causa constricção e aumento da resistência dos vasos pulmonares. As doenças que causam vasoconstricção pulmonar hipóxica por alterações na ventilação (constricção alérgica aguda [asma] ou doença pulmonar crônica) e aumento da resistência como mecanismo compensatório geram conseqüente aumento substancial na pressão arterial pulmonar, condição denominada hipertensão pulmonar (Cunningham, 2004).

De acordo com Ricachinevsky e Amantéa (2006), independente da etiologia associada à presença de HP, algumas alterações encontradas são comuns. Da mesma maneira, dentro de uma mesma doença associada à presença de HP, pode-se observar heterogeneidade das lesões encontradas. Os mesmos autores descrevem que as alterações mais precoces estão situadas no endotélio vascular, fonte importante de mediadores locais que contribuem para o controle do tônus vasomotor e remodelamento estrutural. As alterações funcionais vasculares pulmonares incluem diminuição da produção de substâncias vasodilatadoras e antiproliferativas tais como a prostaciclinas e óxido nítrico (NO) e aumento da produção de substâncias vasoconstritoras e mitógenos como a endotelina. A partir da ocorrência do desequilíbrio destes medidadores, temse consequente aumento do trombo- 
xano e diminuição das prostaciclinas, aumento de endotelina e diminuição do NO.

Estudos realizados em pacientes humanos demonstram que a endotelina (ET-1) é um potente vasoconstritor endógeno produzido primariamente pelas células do endotélio vascular, a qual apresenta importante papel na circulação pulmonar frente à estimulação de dois receptores, a ET-A e ET-B, responsáveis principalmente por gerar proliferação de fibroblastos, depósito de colágeno e vasoconstrição. Em condições patológicas, o aumento da expressão de receptores de ET-B na musculatura lisa e a proliferação de fibroblastos são os principais fatores responsáveis pela potente vasoconstrição e efeitos pró-fibróticos que causam efeitos deletérios como hipertrofia, fibrose e inflamação, sendo estas as principais alterações patológicas observadas em pacientes acometidos por HP. Existem outros mediadores que também podem estar envolvidos tais como a serotonina, fator de crescimento derivado de plaquetas, angiotensina, perda da síntese vascular de NO e prostaciclinas por expressão genética. A lesão endotelial inicial resulta em recrutamento de mediadores vasoativos locais, promovendo um estado pró-coagulante que leva à consequente obstrução vascular. Além disso, alterações nos canais de cálcio e potássio da musculatura lisa da circulação pulmonar também parecem estar envolvidas no início e/ou progressão da HP (O'Callaghan e Gaine, 2004; Ricachinevsky e Amantéa, 2006). Segundo Stepien (2009), os mesmos processos patológicos envolvidos na determinação de HP na medicina são igualmente descritos na medicina veterinária.

A severidade da doença vascular pulmonar pode ser classificada de I a VI de acordo com o grau e aparecimento de hipertrofia da camada média, espessamento celular da camada íntima e/ou espessamento oclusivo da camada íntima, lesões com dilatação vascular, lesões plexiformes e artrite necrosante aguda (Ricachinevsky e Amantéa, 2006). No estudo realizado por Zabka e colaboradores (2006) no Hospital Escola de Medicina Veterinária da Califórnia Daves (VMTH - Veterinary Medical Teaching Hospital), com o objetivo de revisar os achados clínicos e patológicos de 6 cães com diagnóstico de HP ou HP idiopática, os autores demonstraram que todas as formas de $\mathrm{HP}$, independente da causa, possuem uma similaridade quanto ao aspecto das alterações patológicas, incluindo a hipertrofia das artérias e hipertrofia concêntrica do ventrículo direito.

\section{SINAIS CLIINICOS}

Segundo Ricachinevsky e Amantéa (2006), seres humanos com $\mathrm{HP}$, independente da etiologia, não apresentam um quadro clínico específico. As crianças apresentam sintomas predominantes de baixo débito cardíaco, como falha no crescimento, letargia, irritabilidade, taquipnéia e taquicardia. Em contrapartida, os adultos referem algum grau de desconforto respiratório, fadiga, cefaléia, dor torácica, sendo esta provavelmente decorrente de isquemia ventricular direita, e episódios de síncope pósesforço. Eventualmente casos de morte súbita podem ocorrer.

Os pacientes veterinários podem apresentar intolerância ao exercício, angústia respiratória, tosse, estertores, crepitações e expiração prolongada (Kellum e Stepien, 2007), e segundo Hegewald et al. (2007), a dispnéia, ortopnéia, fraqueza, dor torácica e hemoptises podem estar presentes. Zabka e colaboradores (2006) descrevem que os sinais clínicos se apresentam caracterizados por dispnéia ou 
taquipnéia, letargia e intolerância ao exercício, normalmente associados a estresse ou excitação. No entanto, segundo Belerenian (2003) mais de $50 \%$ da área vascular pulmonar deve estar acometida para o surgimento de sinais clínicos.

Hawkins (2004) descreve que a hiperfonese e os desdobramentos da segunda bulha cardíaca podem ser auscultados, assim como sopro e/ou som de galope, sugerindo uma disfunção ventricular direita. Caso ocorra grande dilatação do ventrículo direito, pode-se auscultar sopro da valva tricúspide e/ou pulmonar, além da ocorrência de reflexo hepatojugular e visualização de distensão jugular.

Kellum e Stepien (2007) utilizaram para estudo 22 cães com evidência clínica e ecocardiográfica de HP, sendo observado que os sinais de tosse estavam presentes em $77 \%$ dos casos e esforço respiratório em 35 \%. Episódios de síncope (32\%), letargia (32\%) e intolerância a exercício (23\%) foram os sinais menos comumente observados.

\section{DIAGNÓSTICO}

O exame radiográfico pode oferecer algum auxílio diagnóstico para HP. Apesar de não ser uma ferramenta diagnóstica específica para sua identificação, pode-se observar alterações compatíveis como aumento do tamanho do ventrículo direito, efusão pleural, artérias pulmonares dilatadas e evidência de doença pulmonar subjacente, tais como, fibrose pulmonar, bronquite crônica, embolia pulmonar e colapso traqueobronquial (Hawkins, 2004; Belerenian, 2003). Belerenian (2003) ressalta que as alterações radiológicas no átrio direito são variáveis e pode haver infiltrados alveolares por hemorragia ou inflamação com oclusão de uma artéria maior. Segundo Allen e Mackin (2002), cães com cor pulmonale apresentam indício radiográfico de dilatação do ventrículo direito, aumento do contato esternal na projeção lateral e evidência do componente ventricular direito na silhueta cardíaca, na projeção dorsoventral (o conhecido "D invertido").

$\mathrm{Na}$ eletrocardiografia pode ser observado presença de ondas $P$ pulmonale, sobrecarga ventricular direita (onda S em DI, DII, DIII e aVF), onda Q profunda e eixo com desvio para direita (Belerenian, 2003). O mesmo autor relata que podem aparecer alterações na onda T e no segmento ST em virtude da hipóxia e adiciona que as arritmias e distúrbios de condução são freqüentes. Dos 54 cães avaliados no estudo realizado por Pyle e colaboradores (2004), 40 deles apresentaram alterações eletrocardiográficas como desvio do eixo para a direita, complexo ventricular prematuro, complexo atrial prematuro e fibrilação atrial.

Segundo Morais (2008), a disponibilidade de novas tecnologias de diagnóstico por imagem permite a rápida e fácil identificação da HP em pacientes veterinários. O exame ecocardiográfico com Doppler é um método rápido e não invasivo para o diagnóstico da HP em cães, porém exige habilidade do operador. Hegewald e colaboradores (2007) corroboram com Sarraff-Lopes e colaboradores (2006) afirmando que este exame possibilita estimar a pressão na artéria pulmonar e avaliar a velocidade e quantidade de fluxo regurgitante pulmonar e tricúspide. Segundo Belerenian (2003) e Rovedder e colaboradores (2008), as principais alterações ecocardiográficas observadas são dilatação ou hipertrofia do ventrículo direito, insuficiência da valva tricúspide, movimento septal paradoxal, aumento da artéria pulmonar, diminuição do tamanho do ventrículo esquerdo com função normal e espessamento da parede livre e septo do ventrículo esquerdo. A sobrecarga de volume do ventrículo direito ocorre quando a pressão diastólica aumenta 
levando à insuficiência de tricúspide. $\mathrm{Na}$ HP congênita observa-se hipertrofia concêntrica moderada a grave do ventrículo direito, enquanto que na adquirida há dilatação e hipertrofia do ventrículo direito.

$\mathrm{O}$ diagnóstico definitivo de HP é feito por cateterismo cardíaco, um procedimento invasivo, porém considerado padrão ouro para o diagnóstico e determinação da severidade da doença (Rovedder et al., 2008; Hegewald et al., 2007; Hawkins 2004) ou ecocardiografia com Doppler citada anteriormente (Hegewald et al., 2007; Sarraff-Lopes et al., 2006). Morais (2008) afirma que cães e gatos que apresentam pressão sistólica igual ou superior a $30 \mathrm{mmHg}$ e/ou pressão diastólica igual ou superior a $15 \mathrm{mmHg}$ em artéria pulmonar são consideradas diagnóstico definitivo para HP e, segundo Belerenian (2003), a pressão sistólica igual ou superior a $60 \mathrm{mmHg}$ é considerada responsável pela insuficiência cardíaca congestiva direita. Hawkins (2004) afirma que a HP geralmente é identificada em pacientes veterinários durante a avaliação de animais com doença pulmonar crônica ou insuficiência cardíaca direita.

Segundo Lapa et al. (2006), o diagnóstico de hipertensão arterial pulmonar idiopática só é estabelecido após a realização de todos os exames complementares, afastando as demais condições associadas possíveis.

\section{TRATAMENTO}

O tratamento objetiva a estabilização cardiovascular, melhora da oxigenação e redução da pressão arterial pulmonar (Bentlin et al., 2005). Sempre que possível o tratamento é direcionado à eliminação da causa primária. Para causas respiratórias irreversíveis de HP, como a doença obstrutiva crônica, observou-se diminuição da pressão pulmonar e prolongamento da sobrevivência de seres humanos submetidos à terapia com oxigênio. Em pacientes veterinários, a administração crônica de oxigênio nem sempre é possível, porém, em uma condição aguda, a suplementação de oxigênio é um componente crucial do tratamento que deve ser administrada várias horas por dia conforme a gravidade do caso (Belerenian, 2003).

Segundo Souza e colaboradores (2003), as opções de tratamento são limitadas na HP grave e os estudos recentes da medicina citam o uso da substância sildenafil, um inibidor da 5fosfodiesterase. As fosfodiesterases correspondem a uma superfamília de enzimas que inativam a adenosina e guanosina-monofosfato cíclico, mensageiras de prostaciclina e óxido nítrico, possuindo abundante expressão principalmente no tecido pulmonar (Moraes, 2008). A substância sildenafil é utilizada primordialmente em pacientes acometidos por disfunção erétil, porém também é capaz de reduzir a resistência vascular pulmonar em voluntários sadios com vasoconstrição pulmonar induzida por hipoxemia, humanos com $\mathrm{HP}$ e em animais com HP grave experimental (Souza et al., 2003).

Novos inibidores seletivos da 5fosfodiesterase, vardenafil e tadalafil, com o mesmo mecanismo de ação, porém com propriedades fármacocinéticas próprias, foram igualmente lançados para o tratamento da disfunção erétil, mas poucos estudos existem sobre a sua utilização na HP (Carvalho et al., 2006). Ghofrani e colaboradores (2004) comparam os efeitos hemodinâmicos do vardenafil e tadalafil em relação ao sildenafil na vasculatura pulmonar e sistêmica em pacientes humanos com HP por 120 minutos. Todos os inibidores da 5fosfodiesterase causaram vasodilatação pulmonar significante, mas diferiram no tempo para o seu efeito máximo e na seletividade. $O$ tadalafil obteve seu 
efeito máximo em 75 a 90 minutos (contra 40 a 45 minutos para 0 vardenafil e 60 minutos para 0 sildenafil). Assim, os autores concluíram que tadalafil e sildenafil mostraram maior seletividade para a vasculatura pulmonar (queda no índice resistência vascular pulmonar/ resistência vascular sistêmica) e apenas o sildenafil mostrou melhora na oxigenação arterial. Souza e colaboradores (2003) afirmam que o uso do sildenafil durante 3 meses demonstrou-se seguro e está associado à melhora hemodinâmica e à qualidade de vida em pacientes com HP.

Os antagonistas da endotelina representam uma nova classe farmacológica para o tratamento da HP (O'Callaghan e Gaine, 2004). O bosentan, fármaco antagonista de receptor ET-A e ET-B, atua pela inibição da vasoconstrição, da ação da mitogenicidade e do remodelamento (Ricachinevsky e Amantéa, 2006). Segundo O'Callaghan e Gaine (2004), devido à sua capacidade de bloquear a ação vasoconstritora da endotelina, resulta na melhora na função hemodinâmica cardiopulmonar e ao retardo na progressão da doença. Esta substância tem sido e estudado na medicina e também demonstrou eficácia em animais no estudo experimental realizado por Clozel e colaboradores (2005). Neste estudo, os pesquisadores utilizaram em ratos doses subcutâneas de um alcalóide monocrotalina, extraído da planta Crotalaria retusa, para induzir HP através de inflamação vascular pulmonar aguda. Este estudo tinha como objetivo avaliar os efeitos da monoterapia com bosentan e com sildenafil, e posteriormente avaliou-se a eficácia da associação das duas substâncias. Tanto a terapia isolada de bosentan ou sildenafil demonstrou boa eficácia, porém a associação dos dois fármacos resultou em efeitos adicionais para redução da pressão na artéria pulmonar, reduzindo significativamente a hipertrofia ventricular direita.

A utilização de fármacos vasodilatadores é discutível. Nos cães, o efeito sobre a vasculatura pulmonar é imprevisível, e efeitos colaterais como hipotensão sistêmica e taquicardia podem contribuir para a disfunção cardíaca direita. Por esta razão, doses de teste do fármaco devem ser administradas antes da prescrição do tratamento, enquanto as pressões sangüínea, pulmonar e sistêmica estiverem sendo monitoradas por cateterização cardíaca. A hidralazina, nefedipina prostaciclina, adenosina, óxido nítrico e diltiazen são propostos por Belerenian (2003) e Hawkins (2004). Souza e colaboradores (2003) afirmam que a limitação em relação ao uso de agentes vasodilatadores é atribuída, em parte, à baixa de potência e de seletividade desses medicamentos em relação à circulação pulmonar.

As teofilinas são recomendadas para animais com hipertensão secundária à doença pulmonar crônica. Os benefícios em animais de pequeno porte incluem vasodilatação pulmonar, broncodilatação, aumento da contratilidade do diafragma e diminuição da fadiga dos músculos respiratórios (Hawkins, 2004). Belerenian (2003) descreve o uso da prednisolona por seu efeito antiinflamatório e antifibrótico; ainda acrescenta que a azatioprina, colchicina, pirfenidona, ciclofosfamida e relaxina ainda encontram-se sob investigação de doses adequadas.

\section{PROGNÓSTICO}

O prognóstico depende da identificação e reversibilidade da doença associada e da extensão das lesões nas artérias pulmonares. Deve ser realizado acompanhamento dos animais com radiografias, ecocardiografias e hemogasometria arterial seriados. Estes exames podem fornecer alguma 
indicação da progressão da doença em indivíduos isolados (Hoeper, 2004).

$\mathrm{O}$ estudo realizado por Ghio e colaboradores (2001) demonstrou que a HP leve a moderada trata-se de uma condição frequentemente observada em pacientes humanos portadores de disfunção ventricular esquerda crônica. Porém, os pacientes portadores de disfunção cardíaca direita possuíam pior prognostico em relação às demais condições, em virtude do estabelecimento de HP severa. Em contrapartida, a avaliação de 54 cães portadores de HP, realizada por Pyle e colaboradores (2004), demonstrou que a associação de HP às doenças respiratórias crônicas determinava um pior prognóstico, igualmente pelo estabelecimento de HP severa.

\section{DISCUSSÃO}

A hipertensão pulmonar idiopática é uma doença rara e acomete principalmente mulheres pós-puberdade e homens acima de 36 anos (Zabka et al., 2006). Morais (2008) afirma maior predisposição nos pacientes caninos em comparação aos felinos. Sarraff-Lopes et al. (2006) observam que, apesar da predisposição, a hipertensão pulmonar é rara em cães.

Sabe-se que nenhuma das substâncias utilizadas para o tratamento da HP promove a cura de pacientes humanos e animais, principalmente em casos de HP idiopática, havendo a possibilidade de ocorrer progressão do quadro clínico dos pacientes acometidos por esta doença (Hoeper, 2004). Apesar de todo avanço terapêutico, apenas $50 \%$ dos pacientes apresentam resposta clínica significativa (Bentlin et. al, 2005).

Segundo Bentlin e colaboradores (2005), o uso de inibidores da 5fosfodiesterase como o Sildenafil em modelos animais com HP mostrou-se um vasodilatador pulmonar seletivo sem efeitos na pressão arterial sistêmica, responsável pela redução da resistência vascular pulmonar na forma de monoterapia ou em associação com prostaciclinas ou óxido nítrico. Kellum e Stepien (2007) observaram que embora tenha existido melhora nos sinais clínicos dos pacientes veterinários tratados com Sildenafil, este não diminuiu significativamente o grau de HP nos cães, ou seja, os exames complementares permaneceram inalterados mesmo com a melhora clínica e o aumento da qualidade de vida.

\section{CONCLUSÃO}

A HP ainda é pouco descrita na medicina veterinária, tornando fundamental a investigação desta afecção em pacientes acometidos por doenças pulmonares ou valvares crônicas. Segundo Ricachinevsky e Amantéa (2006) até o momento não existe cura, nem mesmo uma abordagem terapêutica que possa ser recomendada de maneira universal para pacientes humanos com HP. Entretanto, recentes avanços na fisiopatogenia da HP e o surgimento de novos fármacos, com ação vasodilatadora seletiva sobre o leito vascular pulmonar, contribuíram para a melhora da sobrevida desses pacientes. De acordo com Sarraff-Lopes e colaboradores (2006) novas opções de tratamento para pacientes humanos tem sido estendidas para pequenos animais e estão sendo consideradas alternativas viáveis para a HAP em cães.

\section{REFERÊNCIAS}

ALLEN, D. G.; MACKIN, A. Cor Pulmonale. In: TILLEY, L. P.; GOODWIN, J-K. Manual de Cardiologia para Cães e Gatos. 3.ed; Rooca, 2002, p.185-202.

BELERENIAN, G. Hipertensão Pulmonar. In: BELERENIAN, G. C.; MUCHA, C. J.;

CAMACHO, A. A. Afecções Cardiovasculares 
em Pequenos Animais. 1.ed.: Interbook, 2003, p.218-225.

BENTLIN, M.R.; SAITO, A.; LUCA, A.K.C. et al. Sildenafil no tratamento da hipertensão pulmonar após cirúrgica cardíaca. Jornal de Pediatria, v.81, n.2, p.175-178, 2005.

CARVALHO, A.C.; HOVNANIAN, A.L.; FERNANDES, C.J.C.S. et al. Tadalafil para o Tratamento da Hipertensão Arterial Pulmonar Idiopática. Arquivo Brasileiro de Cardiologia, v.87, n.5, p.195-197, 2006.

CLOZEL, M.; HESS, P.; REY, M. et al. Bosentan, Sildenafil, and Their Combination in the Monocrotaline Model of Pulmonary Hypertension in Rats. Experimental Biology and Medicine, v.231, n.6, p.967-973, 2006.

CUNNINGHAM, J. G. As Circulações Sistêmica e Pulmonar. In: Tratado de Fisiologia

Veterinária. 3. ed. Guanabara Koogan, cap. 21., p. 179-187. 2004.

GHIO, S.; GAVAZZI, A.; CAMPANA, C. et al. Independent and additive prognostic value of right ventricular systolic function and pulmonary artery pressure in patients with chronic heart failure. Journal of the American College of Cardiology, v.37, n.1, p.183-188, 2001.

GHOFRANI, H. A.; VOSWINCKEL, R.; REICHENBERGER, F. et al. Differences in hemodynamic and oxygenation responses to three different phosphodiesterase- 5 inhibitors in patients with pulmonary arterial hypertension: a randomized prospective study. Journal of the American College of Cardiology, v.44, n.7, p.1488-1496, 2004.

HAWKINS, E. C. Doenças do Parênquima Pulmonar. In: ETTINGER, S. J.; FELDMAN, E. C. Tratado de Medicina Interna Veterinária. 5. ed.: Guanabara Koogan, p.1120-1151, 2004.

HEGEWALD, M. J.; MARKEWITZ, B.; ELLIOTT, C. G. Pulmonary hypertension: clinical manifestations, classification and diagnosis. International Journal of Clinical Practice, v.61, n.156, p.5-14, 2007.

HOEPER, M. M.; FAULENBACH, C.; GOLPON, $H$. et al. Combination therapy with bosentan and sildenafil in idiopathic pulmonary arterial hypertension. European Respiratory Journal, v.24, n.6, p.1007-1010, 2004.

KELLUM, H. B.; STEPIEN, R. L. Sildenafil Citrate Therapy in 22 Dogs with Pulmonary Hypertension. Journal of Veterinary Internal Medicine, v.21, n.6, p.1258-1264, 2008.

LAPA, M. S.; FERREIRA, E. V. M.; JARDIM, C. et al. Características clínicas dos pacientes com hipertensão pulmonar e dois centros de referência em São Paulo. Revista da Associação Médica Brasileira, v. 52, n.3, p.139-143, 2006.

MORAIS, A. Causes, Diagnosis, and Treatment of Pulmonary Hypertension. In: Proceeding of the Southern European Veterinary Conference, Barcelona, Spain, 2008, p.17-19.

NEWMAN, J. H. Treatment of Primary Pulmonary Hypertension - The Next Generation. The New England Journal of Medicine, v.346, n.12, p.933-935, 2002.

O'CALLAGHAN, D.; GAINE, S. P. Bosentan: A novel agent for the treatment of pulmonary arterial hypertension. International Journal of Clinical Practice, v.58, n.1, p.69-73, 2004.

PETROS, A. J.; PIERCE, C. M. The management of pulmonary hypertension. Pediatric Anesthesia, v.16, n.8, p.816-821, 2006.

PYLE, R. L.; ABBOTT, J.; MACLEAN, H. Pulmonary Hypertension and Cardiovascular Sequelae in 54 dogs. Journal of Applied Research in Veterinary Medicine, v.2, n.2, p.99-109, 2004.

RICACHINEVSKY, C.P.; AMANTÉA, S.L. Treatment of pulmonary arterial hypertension. Jornal de Pediatria, v.82, n.5, p.153-165, 2006.

ROVEDDER, P.M.E.; ZIEGLER, B.; PINOTTI, A.F.F. et al. Prevalência de hipertensão pulmonar avaliada por ecocardiografia Doppler em uma população de pacientes adolescentes e adultos com fibrose cística. Jornal Brasileiro de Pneumologia, v.34, n.2, p.83-90, 2008.

SARRAFF-LOPES, A.; MOREIRA, T. M. R.; GANDRA, M. et al. Sildenafil no tratamento da hipertensão pulmonar primária em um cão relato de caso. In: XXVII Congresso Brasileiro da Anclivepa e III Congresso da FIAVAC, Vitória, Espírito Santo, Brasil, 2006, 35p.

SOUZA, E.J.R.S.; GARIB, J.R.; GARIB, N.M. et al. Sildenafil for treatment of pulmonary hypertension in association with systemic lúpus erythematosus and anti-phospholipid syndrome. Jornal Brasileiro de Pneumologia, v.29, n.5, p.302-304, 2003.

STEPIEN, R. L. Pulmonary arterial hypertension secondary to chronic left-sided cardiac dysfunction in dogs. Journal of Small Animal Practice, v.50, n.1, p.34-43, 2009.

ZABKA, T.S.; CAMPBELL, F.E.; WILSON, D.W. Pulmonary Arteriopathy and Indiopatic Pulmonary Arterial Hypertension in Six Dogs. Veterinary Pathology, v.43, n.4, p.510-522, 2006. 